\title{
Partition function of the two-dimensional nearest neighbour Ising models for finite lattices in a non-zero magnetic field ${ }^{\#}$
}

\author{
M VINOTHKUMAR, G NANDHINI and M V SANGARANARAYANAN* \\ Department of Chemistry, Indian Institute of Technology Madras, Chennai 600 036, India \\ e-mail: sangara@iitm.ac.in
}

\begin{abstract}
The partition function for two-dimensional nearest neighbour Ising model in a non-zero magnetic field have been derived for a finite square lattice of 16, 25, 36 and 64 sites with the help of graph theoretical procedures, show-bit algorithm, enumeration of configurations and transfer matrix methods.
\end{abstract}

Keywords. Two-dimensional Ising model; partition function; graph theory; transfer matrix; show-bit algorithm.

\section{Introduction}

The analysis of Ising models constitutes a central theme in statistical mechanics and its applicability ranges from the study of critical behaviour of ferromagnet ${ }^{1}$ to temperature-dependence of base pair sequences in DNA. ${ }^{2}$ While the one-dimensional nearest neighbour Ising model did not exhibit phase transitions, the exact solution of the two-dimensional Ising model for zero magnetic field reported by Onsager ${ }^{3}$ yielded an explicit expression for critical temperatures. Lee and $\mathrm{Yang}^{4}$ provided a complete solution of the two-dimensional Ising model for vanishing magnetic fields, validating the exact solution of Onsager. However, subsequent attempts of solving the two-dimensional Ising models when the magnetic field is non-zero has remained elusive till now, although methodologies employing Bragg-Williams approximation, ${ }^{5}$ Bethe ansatz, ${ }^{6}$ series expansions, ${ }^{7}$ renormalization group, ${ }^{8}$ scaling hypothesis, ${ }^{9}$ etc. have been investigated. In view of its equivalence with binary alloys and lattice gas description of fluids, ${ }^{10}$ the results derived in the context of Ising models become applicable mutatis mutandis to various topics in solid state and condensed matter physics. The two-dimensional Ising model ${ }^{11}$ finds applicability in the study of order-disorder transitions, ${ }^{12}$ electrochemical interfaces, ${ }^{13}$ phase separation in self-assembled monolayer films ${ }^{14}$ protein folding, ${ }^{15}$ free energies of surface steps ${ }^{16}$ etc. As pointed out elsewhere ${ }^{17}$ 'In terms of the number of papers published, Ising model ranks as probably the most celebrated model in mathematical physics.'

\footnotetext{
\#Dedicated to Prof. N Sathyamurthy on his 60th birthday

*For correspondence
}

In this communication, we derive the canonical partition function of the two-dimensional nearest neighbour Ising models for a non-zero magnetic field pertaining to a square lattice of 16 sites using (i) enumeration of all the $2^{16}$ configurations and the corresponding energies; (ii) 'show-bit' algorithm (decimal to binary conversion); (iii) graph theoretical approach of counting of 'black-white' edges and (iv) transfer matrix formulation. The transfer matrix method is employed for estimating the partition function for $N=64$.

\section{Methodology}

\subsection{Partition function for a square lattice of 16 sites}

Consider the two-dimensional nearest neighbour Ising model on a square lattice ${ }^{18}$ with the Hamiltonian given by

$$
\begin{aligned}
H_{T}= & -J \sum_{<i j>}\left(\sigma_{i, j} \sigma_{i, j+1}+\sigma_{i, j} \sigma_{i+1, j}\right) \\
& -H \sum \sigma_{i, j},
\end{aligned}
$$

where $J$ is the nearest neighbour interaction energy, $H$ being the external magnetic field, $i$ and $j$ denote the row and column index, respectively. When the periodic boundary conditions are assumed the corresponding canonical partition function is defined as ${ }^{19}$

$$
Q(H, T)=\sum_{i=1}^{2^{N}} e^{-\frac{\left(H_{T}\right)_{i}}{k T}},
$$

where $k$ denotes the Boltzmann constant, $T$ being the absolute temperature. 
By denoting $E_{i}$ as the energy of $i$ th configuration, the partition function is re-written as

$$
Q(H, T)=\sum_{i=1}^{2^{N}} e^{-\frac{E_{i}}{k T}},
$$

where $E_{i}$ refers to the energy of $i$ th configuration and $i$ varies from 1 to $2^{N}$.

For brevity, we represent $e^{J / k T}$ as $x$ and $e^{H / k T}$ as $y ; J$ and $H$ are assumed to be positive quantities. For a square lattice of 16 sites, our tour de force consists in deducing the partition function by systematically enumerating all the 65,536 configurations; this task is accomplished by employing Visual Basic programming in conjunction with the Excel software. The energies are typically obtained from the formula

$$
\begin{aligned}
& {\left[\sigma_{1,1} \times\left(\sigma_{1,2}+\sigma_{2,1}\right)+\sigma_{1,2} \times\left(\sigma_{1,3}+\sigma_{2,2}\right)+\sigma_{1,3}\right.} \\
& \times\left(\sigma_{1,4}+\sigma_{2,3}\right)+\sigma_{1,4} \times\left(\sigma_{2,4}+\sigma_{1,1}\right)+\sigma_{2,1} \\
& \times\left(\sigma_{2,2}+\sigma_{3,1}\right)+\sigma_{2,2} \times\left(\sigma_{2,3}+\sigma_{3,2}\right)+\sigma_{2,3} \\
& \times\left(\sigma_{2,4}+\sigma_{3,3}\right)+\sigma_{2,4} \times\left(\sigma_{2,1}+\sigma_{3,4}\right)+\sigma_{3,1} \\
& \times\left(\sigma_{3,2}+\sigma_{4,1}\right)+\sigma_{3,2} \times\left(\sigma_{3,3}+\sigma_{4,2}\right)+\sigma_{3,3} \\
& \times\left(\sigma_{3,4}+\sigma_{4,3}\right)+\sigma_{3,4} \times\left(\sigma_{4,4}+\sigma_{3,1}\right)+\sigma_{4,1} \\
& \times\left(\sigma_{4,2}+\sigma_{1,1}\right)+\sigma_{4,2} \times\left(\sigma_{4,3}+\sigma_{1,2}\right)+\sigma_{4,3} \\
& \left.\times\left(\sigma_{4,4}+\sigma_{1,3}\right)+\sigma_{4,4} \times\left(\sigma_{4,1}+\sigma_{1,4}\right)\right] \\
& +\left\{\sigma_{1,1+} \sigma_{2,1+} \sigma_{3,1+} \sigma_{4,1+} \sigma_{2,1+} \sigma_{2,2+} \sigma_{2,3+} \sigma_{2,4+}\right. \\
& \left.\sigma_{3,1+} \sigma_{3,2+} \sigma_{3,3+} \sigma_{3,4+} \sigma_{4,1+} \sigma_{4,2+} \sigma_{4,3+} \sigma_{4,4}\right\},
\end{aligned}
$$

wherein the periodic boundary conditions are incorporated. All the 'energy terms' can be obtained by taking into account various configurational arrangements. The $\operatorname{sum} \sum_{i=1}^{65536} e^{-\frac{E_{i}}{k T}}$ yields the canonical partition function for the square lattice of 16 sites as

$$
\begin{aligned}
\mathrm{Q}_{16}= & x^{32}\left(y^{16}+y^{-16}\right)+16 x^{24}\left(y^{14}+y^{-14}\right) \\
& +\left(88 x^{16}+32 x^{20}\right)\left(y^{12}+y^{-12}\right) \\
& +\left(96 x^{16}+256 x^{12}+208 x^{8}\right)\left(y^{10}+y^{-10}\right) \\
& +\left(24 x^{16}+256 x^{12}+736 x^{8}+576 x^{4}+228\right) \\
& \times\left(y^{8}+y^{-8}\right)+\left(192 x^{12}+688 x^{8}+1664 x^{4}\right. \\
& \left.+448 x^{-4}+128 x^{-8}+1248\right)\left(y^{6}+y^{-6}\right) \\
& +\left(96 x^{12}+704 x^{8}+1824 x^{4}+1568 x^{-4}\right. \\
& \left.+768 x^{-8}+64 x^{-12}+56 x^{-16}+2928\right) \\
& \times\left(y^{4}+y^{-4}\right)+\left(64 x^{12}+624 x^{8}\right. \\
& +1920 x^{4}+3136 x^{-4} 1392 x^{-8}+512 x^{-12} \\
& \left.+96 x^{-16}+16 x^{-24} 3680\right)\left(y^{2}+y^{-2}\right)
\end{aligned}
$$

$$
\begin{aligned}
& +\left(2 x^{-32}+64 x^{-20}+120 x^{-16}+576 x^{-12}\right. \\
& +2112 x^{-8}+3264 x^{-4}+1600 x^{4}+768 x^{8} \\
& \left.+8 x^{16}+4356\right)
\end{aligned}
$$

where $x=e^{J / k T}$ and $y=e^{H / k T}$. The coefficient of the ' $H$ ' term viz., the summation of the spin variables occurs within the curly bracket of equation 3 .

Although the lattice size is indeed very small, the cumbersome manner in which the coefficients appear provide a clue to the complexity for higher values of $N$.

Furthermore, this 'brute-force' enumeration becomes tedious as $N$ increases.

\subsection{Show-bit algorithm}

An alternate method amenable for easier computation referred to as 'Show-bit' algorithm can also be employed for small values of $N$. In this algorithm, a decimal number is converted to its corresponding binary number. The energy of each configuration is estimated and progressively summed up in order to compute the corresponding partition function for each $N$. The partition functions for $N=16$ deduced from this method are entirely in agreement both numerically and analytically with that obtained from equation 4. A Message Passsing Interface (MPI) program written in $\mathrm{C}++$ enables the estimation of the partition function for $N=25$ and $N=36$ using the above approach with less time. However, this method also becomes very involved $^{20}$ for $N>36$.

\subsection{Graph-theoretical approach}

The connection of the Ising model problem to the combinatorial graph theory is well known. ${ }^{21}$ The essential premise in this context is that the two-dimensional Ising model can be visualized as a bipartite graph $G$ consisting of the set of vertices $V$ and edges $E$ viz. $G=\{V, E\}$. The vertices represent the arrangement of +1 ('black') and -1 ('white') spins and the edges correspond to the connectivity between the vertices. The black-white edges correspond to the perfect matching of the graph. The number of ways in which a black-white edge can arise, correspond to its weight. This strategy is analogous to the weighted graph colourings problem wherein adjacent vertices have different colours. $^{22}$

For brevity, let ' $p$ ' denote the number of +1 spins (black vertex) and ' $q$ ' represent the black-white edge. Thus, the estimation of the partition function in a nonzero magnetic field is now transformed as counting of the black-white edges of a square lattice of $N$ sites 
wherein adjacent and non-adjacent black-white edges along with their identities need to be included, taking into account, the periodic boundary conditions. As an illustration for a square lattice of $N$ sites, the number of black sites may be denoted as ' $p$ ' and hence that of white sites is $N-p$. Let ' $q$ ' be the number of blackwhite edges formed. Then, $A(p, q)$ denotes the number of times a configuration occurs with ' $p$ ' number of black sites and ' $q$ ' number of black-white edges. For example, when there are two adjacent black sites, the number of black-white edges will be six, incorporating the periodic boundary conditions, although the two black sites can occur at any place among the four rows and four columns (for $N=16$ ).

The value of ' $p$ ' may vary from 0 to $N$ since there can be no black sites, 1 black site, 2 black sites....N black sites etc. Accordingly, the value of ' $q$ ' also changes. The number of black-white edges $(q)$ changes from 0 to $2 \mathrm{~N}$ and is also dictated by the value of $p$, since for a given value of $p$, the black sites can be adjacent or non-adjacent. For example, if $p=3$ (3 black sites), the following cases arise: (i) all the three can be adjacent, (ii) two of them can be adjacent and the remaining site is present elsewhere within the square and (iii) all the three may be present randomly. Table 1 denotes $A(p, q)$ values for a square lattice of 16 sites deduced using a program written in $\mathrm{C}++$ and the Appendix A lists the $\mathrm{C}++$ code for the same.

The number of black-white edges $A(p, q)$ is related to the canonical partition function in two-dimensions as ${ }^{23}$

$$
Q(x, y)=\sum_{p, q} A(p, q) x^{2(N-q)} y^{N-2 p},
$$

where $x=e^{J / k T}$ and $y=e^{H / k T}$ as before. Incorporating $A(p, q)$ and the corresponding $p$ and $q$ values for $N=16$, the partition function is derived for $N=16$. This equation is identical with that arising from the other two approaches mentioned earlier. The estimation of $A(p, q)$ is extended for $N=25$ and $N=36$ in an analogous manner. This enumeration method for counting of vertices and edges has tremendous potentialities and among them, mention may be made of the following: protein folding ${ }^{24}$ stability of polycyclic benzenoid hydrocarbons, ${ }^{25}$ organization of biochemical networks, ${ }^{26}$ percolation on two-dimensional lattices ${ }^{27}$ etc.

\subsection{Transfer matrix formulation}

For one-dimensional Ising models, the basic transfer matrix turns out to be $2 \times 2$, whose eigenvalues can then be employed for all lattice sizes. This simplicity is no longer present for the two-dimensional case. Furthermore, for a square lattice of $n^{2}$ sites, the transfer matrix ${ }^{28}$ is of dimension $2^{n} \times 2^{n}$ where $n$ is the number of rows or columns, thus implying that the matrix needs to be constructed separately for each lattice size in general.

It is of interest to point out that the procedure of Kramers and Wannier ${ }^{29}$ for two-dimensional Ising models results in a double-shifted circulant matrix and is valid irrespective of the lattice size albeit at $H=0$. Inspired by this rigorous methodology, it is tempting to formulate a matrix version for $H \neq 0$ too. In the case of one-dimensional Ising models, a Toeplitz matrix does

Table 1. $A(p, q)$ values which arise from the counting of 'black-white' edges for a square lattice of 16 sites leading to the partition function. For $p=0$ and $16(q=0)$ the coefficient is 1 and for $p=1$ and $15(q=4)$ the coefficient is 16 (not shown in the table for clarity).

\begin{tabular}{|c|c|c|c|c|c|c|c|c|c|c|c|c|c|}
\hline$q \backslash p$ & 2 & 3 & 4 & 5 & 6 & 7 & 8 & 9 & 10 & 11 & 12 & 13 & 14 \\
\hline 6 & 32 & & & & & & & & & & & & 32 \\
\hline 8 & 88 & 96 & 24 & & & & 8 & & & & 24 & 96 & 88 \\
\hline 10 & & 256 & 256 & 192 & 96 & 64 & 0 & 64 & 96 & 192 & 256 & 256 & \\
\hline 12 & & 208 & 736 & 688 & 704 & 624 & 768 & 624 & 704 & 688 & 736 & 208 & \\
\hline 14 & & & 576 & 1664 & 1824 & 1920 & 1600 & 1920 & 1824 & 1664 & 576 & & \\
\hline 16 & & & 228 & 1248 & 2928 & 3680 & 4356 & 3680 & 2928 & 1248 & 228 & & \\
\hline 18 & & & & 448 & 1568 & 3136 & 3264 & 3136 & 1568 & 448 & & & \\
\hline 20 & & & & 128 & 768 & 1392 & 2112 & 1392 & 768 & 128 & & & \\
\hline 22 & & & & & 64 & 512 & 576 & 512 & 64 & & & & \\
\hline 24 & & & & & 56 & 96 & 120 & 96 & 56 & & & & \\
\hline 26 & & & & & & 0 & 64 & 0 & & & & & \\
\hline 28 & & & & & & 16 & 0 & 16 & & & & & \\
\hline 30 & & & & & & & 0 & & & & & & \\
\hline 32 & & & & & & & 2 & & & & & & \\
\hline
\end{tabular}


exist $^{30}$ for $H \neq 0$ and $J \neq 0$ which can be obtained from the Discrete Fourier Transformation. ${ }^{31}$ However, for two-dimensional Ising models, the methodology for constructing such Toeplitz matrices for $N \geq 16$ is quite tedious; nevertheless this task when accomplished renders it possible to exploit well-known theorems pertaining to asymptotic limits for eigenvalues of circulant matrices. ${ }^{32}$ Thus, the thermodynamic limit of $N \rightarrow \infty$ for non-zero magnetic field may possibly be reached from the eigenvalues of the circulant matrices. If the correct matrix for a square lattice of $N$ sites is constructed, the partition function follows from

$$
Q(H, T)=\sum_{i=1}^{2^{n}}\left(\lambda_{i}\right)^{n},
$$

where $n$ denotes the number of rows or columns.
While there are 65,536 configurations for a square lattice of $4 \times 4$ sites, the transfer matrix is indeed a $16 \times 16$ matrix consisting of 256 elements. The ferreting out of 256 elements out of the entire set of 65,536 elements in order to construct the transfer matrix appears at first sight to be daunting. However, as has been demonstrated by Kramers and Wannier ${ }^{29}$ there exists an inherent symmetry of the two-dimensional Ising models which can be exploited. Out of the $2^{N}$ configurations, only those configurations which have reflection symmetry are constituents of the matrix. If the condition, site (i) $=$ site $(N / 2+i)$ is satisfied in a configuration, the configuration is said to obey the reflection symmetry. For example, in a square lattice of 16 sites, among all the 65536 elements, only 256 elements satisfy this reflection symmetry property and become the constituents of the matrix, i.e., only those

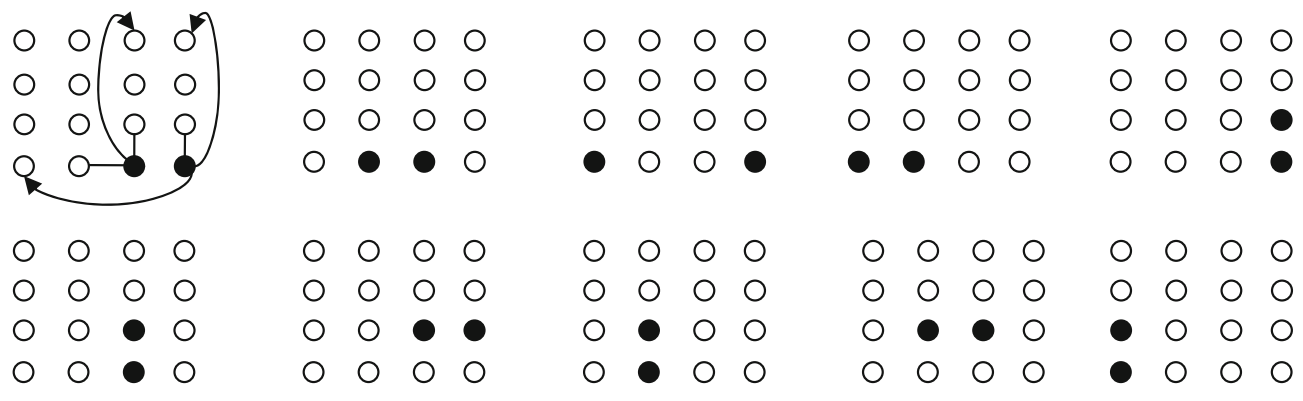

$\begin{array}{llllllllllllllllllll}0 & 0 & 0 & 0 & 0 & 0 & 0 & 0 & 0 & 0 & 0 & 0 & 0 & 0 & 0 & 0 & 0 & 0 & 0 & 0 \\ 0 & 0 & 0 & 0 & 0 & 0 & 0 & 0 & 0 & 0 & 0 & 0 & 0 & 0 & 0 & 0 & 0 & 0 & 0 & 0 \\ 0 & 0 & 0 & 0 & 0 & 0 & 0 & 0 & 0 & 0 & 0 & 0 & 0 & 0 & 0 & 0 & 0 & 0 & 0 & 0 \\ 0 & 0 & 0 & 0 & 0 & 0 & 0 & 0 & 0 & 0 & 0 & 0 & 0 & 0 & 0 & 0 & 0 & 0 & 0 & 0\end{array}$

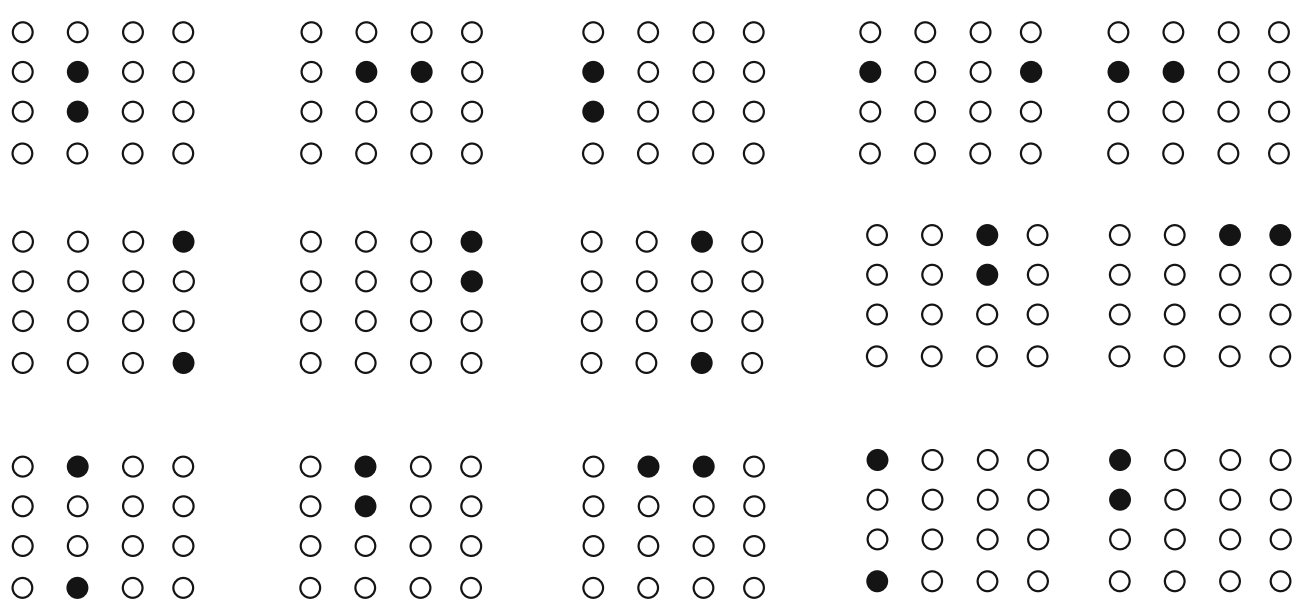

$\begin{array}{llllllll}- & 0 & 0 & 0 & 0 & 0 & 0 & 0 \\ 0 & 0 & 0 & 0 & 0 & 0 & 0 & 0 \\ 0 & 0 & 0 & 0 & 0 & 0 & 0 & 0 \\ 0 & 0 & 0 & 0 & 0 & 0 & 0 & 0\end{array}$

Figure 1. A typical arrangement of the black-white edges $A(p, q)$ for a square lattice of 16 sites. 
configurations, wherein $\operatorname{site}(1)=\operatorname{site}(9)$, site $(2)=$ site(10) and site $(8)=\operatorname{site}(16)$ etc. satisfy the reflection symmetry and constitute the elements of the matrix. As an illustration, consider the following arrangement (figure 1).

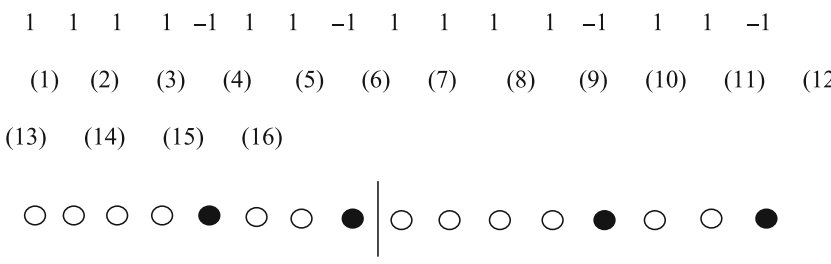

The energy pertaining to the above configuration is $E_{s}=-8 J-8 H$.

Hence, $\exp \left(-E_{s} / k T\right)=\exp (8 J / k T+8 H / k T)=$ $x^{8} y^{8}$. Subsequently, equation 3 is employed to obtain all the coefficients of $J$ and $H$. After calculating the energies of all the 256 reflective symmetric configurations and thus the corresponding expressions, the first 16 elements are arranged in the first row, the next 16 in the second row and so on, till the last 16 elements constitute the last row, thus leading to a $16 \times 16$ matrix. The matrix is such that the upper and lower triangular portions of the main diagonal are symmetric. In view of the matrix being formulated from the 256 elements, the energies $x$ and $y$ need to be scaled as $x_{1}=x^{1 / \mathrm{n}}$ and $y_{1}=y^{1 / \mathrm{n}}$. It should be emphasized that for a lattice of 16 sites, if all the $2^{16}$ elements are arranged as a $2^{8} \times 2^{8}$ matrix, the 256 diagonal elements are same as the 256 matrix elements which arise from the reflection symmetry method. This is obvious since the partition function is the trace of the matrix corresponding to the Hamiltonian. The matrix pertaining to this lattice is provided in figure 2.

When $H=0$, i.e. $y=1$, this matrix will correspond to the zero magnetic field case. Thus, it follows that the matrix for a square lattice of 16 sites pertaining to the two-dimensional nearest neighbour Ising model for a non-zero magnetic field along with the algebraic expression for the corresponding partition function is not unattainable! The partition function equation deduced from the matrix is identical with that arising from the approaches outlined in subsections 2.1, 2.2 and 2.3.

When $N$ is an odd number, this reflection property method becomes inapplicable and hence $Q$ could not be obtained for $N=25$ and $N=49$ using this procedure. As an illustration, the analysis of $N=64$ is considered and in this case, the $2^{64}$ elements are first arranged into a $2^{32} \mathrm{X} 2^{32}$ matrix, which is further reduced to $2^{16} \mathrm{X} 2^{16}$ using the diagonal elements of the former and then expressed as the $2^{8} \mathrm{X} 2^{8}$ using $\mathrm{C}++$ programming. We emphasize that the matrix methodology has yielded the partition functions for $N=64$ in the presence and absence of the magnetic field. The computed partition functions for $N=64$ are in

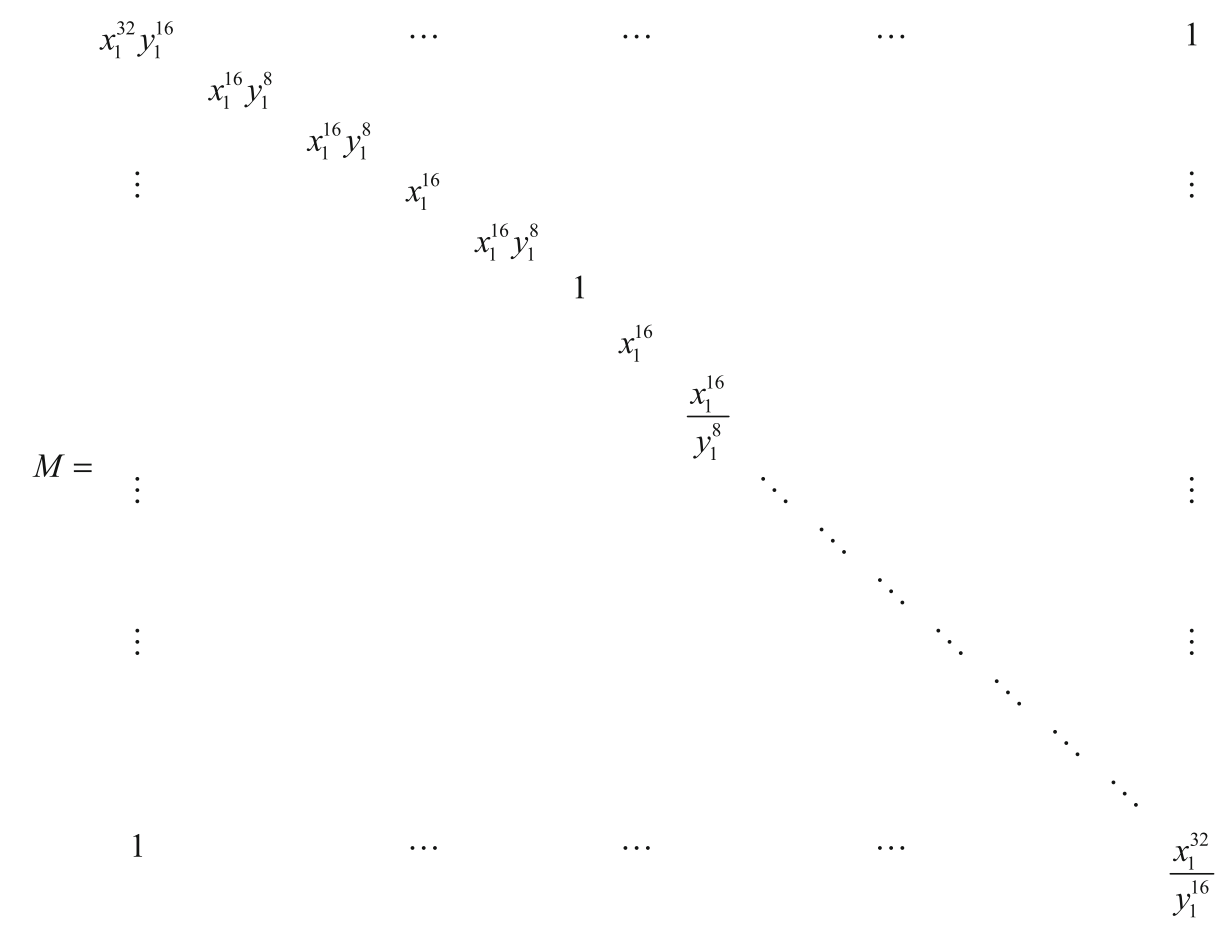

Figure 2. The matrix constructed using the reflection symmetry principle for the square lattice of 16 sites for the two-dimensional Ising model in the presence of the magnetic field. 


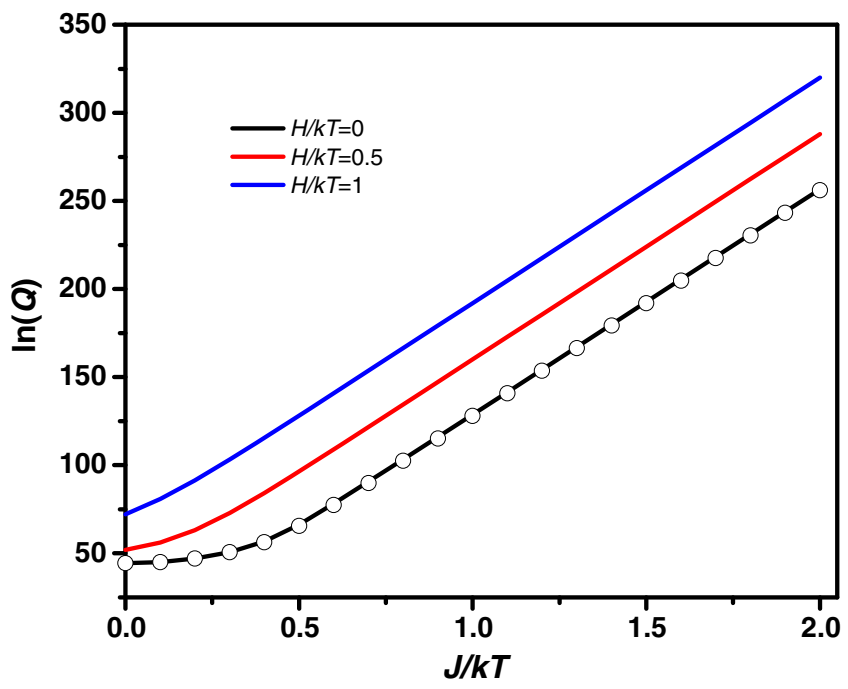

Figure 3. The computed partition functions for the square lattice of 64 sites at various values of $H$ using the $256 * 256$ matrix. For $H=0$, the points denote the values predicted by the Onsager's exact solution while the line arises from equation 6 employing eigenvalues of the $256 \times 256$ matrix.

excellent agreement with Onsager's exact solution for $H=0$ (figure 3). However, this procedure too requires an improvisation depending upon the value of $N$ under consideration.

It appears that the transfer matrix method alone is capable of obtaining the partition function when the lattice size increases; in contrast, the 'show-bit' algorithm is unable to handle large lattices in view of the computational complexity. Appendix B provides the $\mathrm{C}++$ code for the 'show-bit' algorithm in the case of $N=16$.

\section{Thermodynamic quantities}

Having estimated the partition functions for various finite lattices, it is of importance to obtain the thermodynamic quantities such as internal energy, specific heat and magnetization from the corresponding partition function of the respective lattices. Since magnetization for finite lattices is zero, it is not considered in the present study.

It is tempting to postulate ad hoc expressions for the partition functions for infinite lattices, being armed with the corresponding estimates for the lattices of sizes $N=16 ; N=25 ; N=36$ and $N=64$. We refrain from doing so here since these will be of little utility in the absence of results for larger lattices typically at least up to $N=256$. The dimensionless Helmholtz free energy at the critical temperature and $H=0$ has been deduced as -0.92969 by Onsager. ${ }^{3}$

However, the specific heat constitutes a sensitive test for validating any computational procedure. The specific heat is related to the partition function as

$$
\begin{aligned}
U & =k T^{2} \frac{\partial \ln Q}{\partial T} \\
C_{v} & =\frac{\partial U}{\partial T} .
\end{aligned}
$$

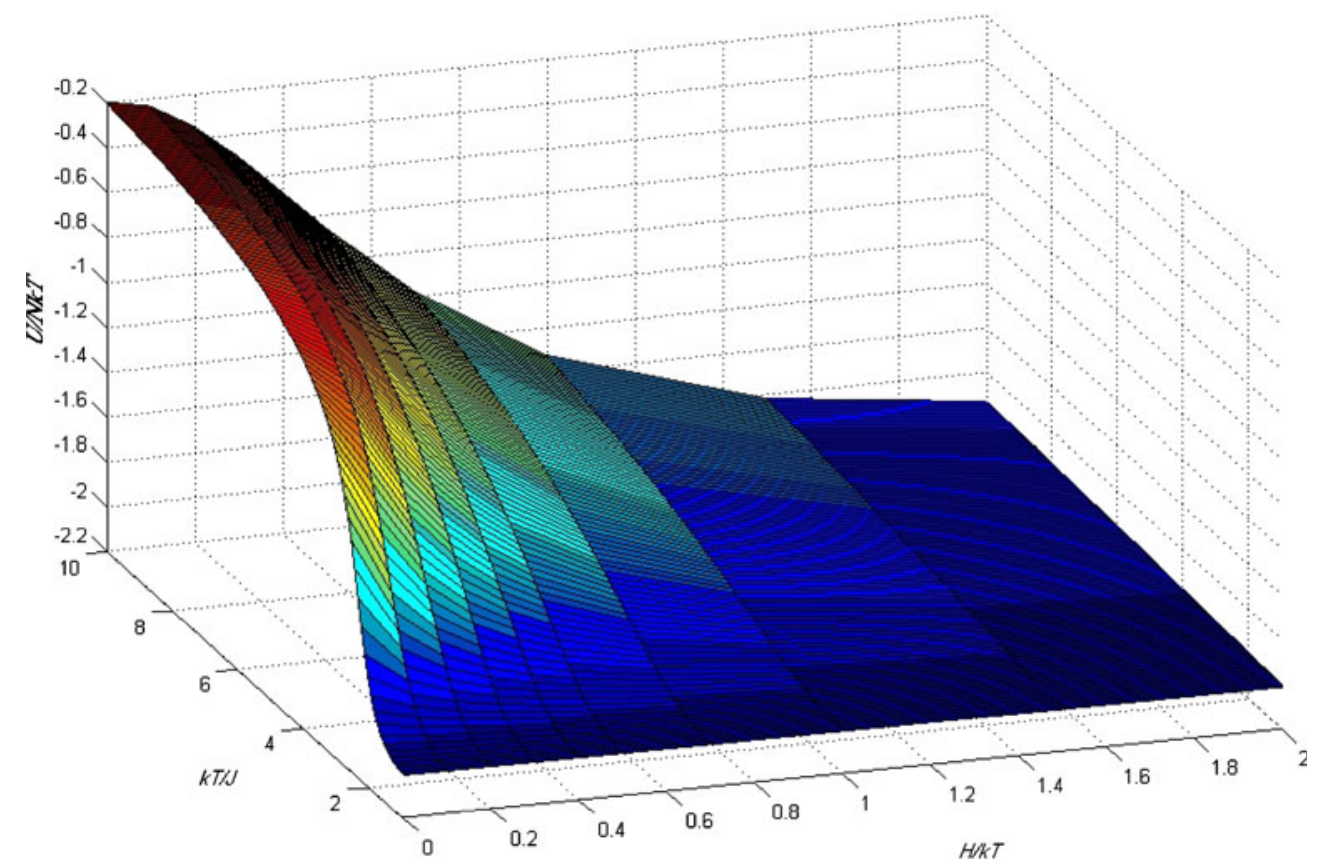

Figure 4. The dependence of the dimensionless internal energy $(U / N k T)$ on various nearest neighbour interaction energies and magnetic fields for $N=64$. 


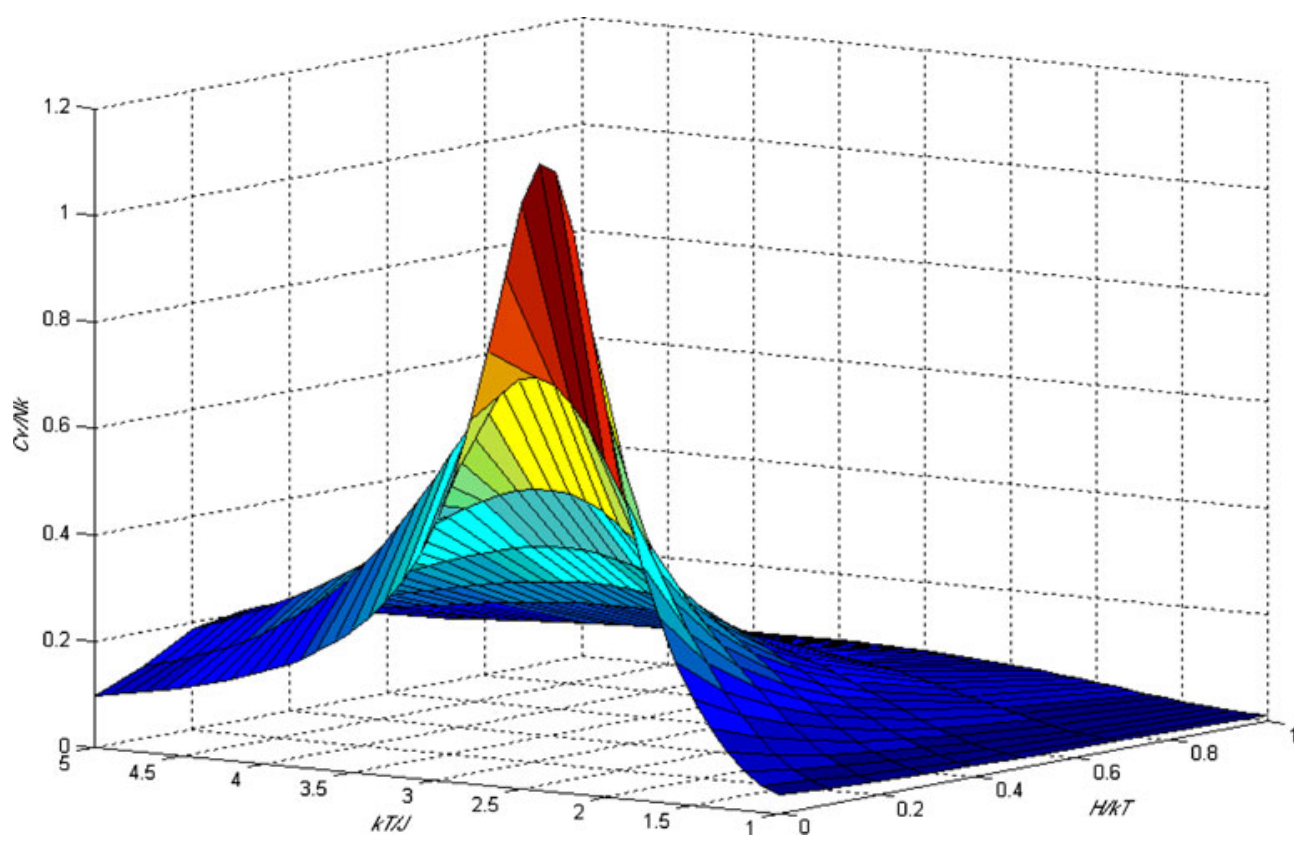

Figure 5. The dependence of the dimensionless specific heat $\left(C_{v} / N k\right)$ on various nearest neighbor interaction energies and magnetic fields for a square lattice of 64 sites.

For clarity, we focus the attention on the variation of the internal energy and specific heat for the square lattice of 64 sites. Figure 4 depicts the dimensionless internal energy for various magnetic fields and nearest neighbour interaction energies. The asymptotic limit for the internal energy exists for infinite sized lattices albeit at the critical temperature viz. $\left(-U_{c} / N k T_{c}=1.414\right)$; however, no such limits have been known for non-zero magnetic fields.

While the variation of $C_{v}$ with ' $H$ ' and ' $J$ ' for 16 and 36 sites can be obtained by analytical differentiation of the partition function expressions, the same for pertaining to $8 \times 8$ lattice needs to be estimated by numerical differentiation of the partition function arising from the transfer matrix method. Figure 5 depicts the predicted variation of $C v$ for the square lattice of 64 sites. The specific heat estimates for 64 sites arising from figure 5 when $H=0$, are in complete agreement with the values arising from the corresponding Monte Carlo simulation values. ${ }^{33}$ It is essential to emphasize that for the zero-magnetic field case, the specific heat and susceptibilities ${ }^{33}$ for values infinite sized lattice exist that are obtained by extrapolation procedures using estimates for small finite sizes.

\section{Perspectives and summary}

The foregoing analysis demonstrates that the partition function for a square lattice of 16 sites can be obtained by different methods in the presence of a magnetic field. However, difficulties emerge for the next square lattice viz., $N=25,36,49,64 \ldots$. In such cases, the matrix method is more suitable although a closed form expression for the partition function is not easy to obtain. Although the lattice sizes employed here are small, further extrapolation procedures may enable the prediction of properties such as internal energy, specific heat, magnetization and susceptibility of the infinite lattice both in the presence and absence of the magnetic field. ${ }^{33}$ While it is true that the Monte Carlo simulation procedures can yield parameters of interest for the two-dimensional Ising models pertaining to very large lattice sizes, the present strategy emphasizes diverse procedures to obtain the partition function itself. Furthermore, these partition functions are exact estimates arising from direct enumeration. The extension of these results to $N \rightarrow \infty$ is not unsurmountable; but will require explicit expressions for at least three more lattice sizes so as to obtain reliable insights. This inter alia requires computations spanning several months using high-end computing machines.

In Summary, the partition functions for twodimensional nearest neighbour Ising models have been computed for square lattice of $N$ sites $(N=16 ; 25 ; 36$; $64)$, in the presence of a magnetic field. The transfer matrix method is suggested as the most suitable method for large values of $N$. The dependence of the internal energy and specific heat on nearest neighbour interaction energies for various magnetic fields is provided for a square lattice of 64 sites. 


\section{Acknowledgments}

The helpful comments of the reviewers are gratefully acknowledged. This work was supported by the Department of Science and Technology (DST), Government of India. The authors thank Dr. A Muthukrishnan for encouragement throughout the course of this work.

\section{Appendix A}

In this Appendix, the $\mathrm{C}++$ Code for computing the black-white edges is provided.

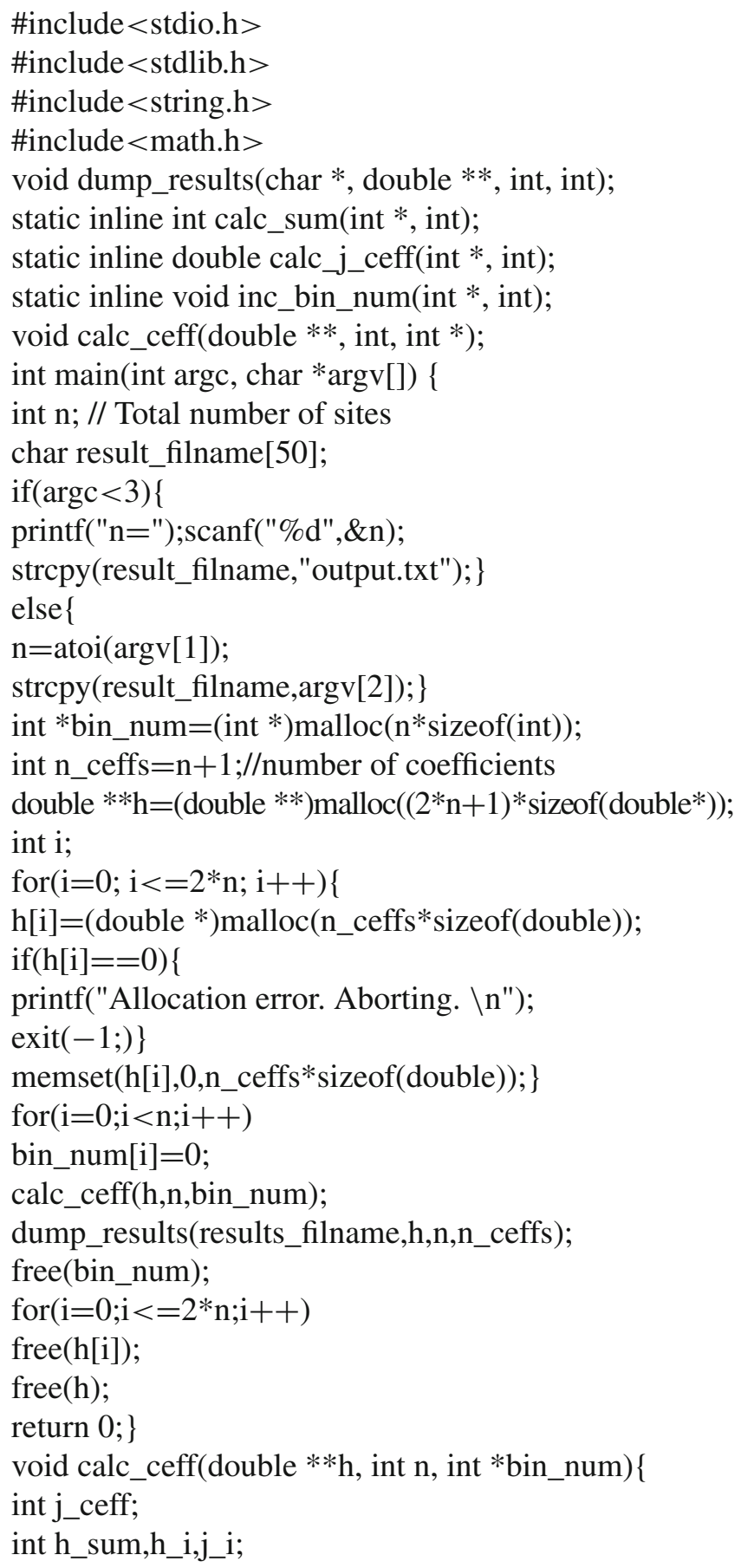

int $\mathrm{i}, \mathrm{j}$;

while(1)\{

$$
\begin{aligned}
& \text { j_ceff }=(\text { calc_j_ceff(bin_num,n })) \text {; } \\
& \text { h_sum }=\text { calc_sum(bin_num,n); } \\
& \mathrm{h} \_\mathrm{i}=\mathrm{h} \_ \text {sum }+\mathrm{n} \text {; } \\
& \mathrm{j} \_\mathrm{i}=\left(\mathrm{j}_{-} \text {ceff }+2 * \mathrm{n}\right) / 4 \text {; } \\
& \mathrm{h}\left[\mathrm{h} \_\mathrm{i}\right]\left[\mathrm{j} \_\mathrm{i}\right]=\mathrm{h}\left[\mathrm{h} \_\mathrm{i}\right]\left[\mathrm{j} \_\mathrm{i}\right]+1 \text {; } \\
& \text { inc_bin_num(bin_num,n); } \\
& \text { for }(\mathrm{i}=0 ; \mathrm{i}<\mathrm{n} \& \& \text { bin_num }[\mathrm{i}]==0 ; \mathrm{i}++) \text {; } \\
& \text { if }(\mathrm{i}==\mathrm{n}) \text { break } ;\} \text { \} }
\end{aligned}
$$

int print_bin_num(int *bin_num,int $\mathrm{n})\{$

int $\mathrm{i}$;

$$
\begin{aligned}
& \text { for }(\mathrm{i}=0 ; \mathrm{i}<\mathrm{n} ; \mathrm{i}++) \\
& \operatorname{printf}(" \% \mathrm{~d} \text { ",bin_num[i]); } \\
& \text { printf("\} \backslash \mathrm { n } " ) ; \} }
\end{aligned}
$$

static inline double calc_j_ceff(int *bin_num, int n)\{

int $\mathrm{m}=$ (int) sqrt(n),i,j;

int *bin_num_cp=(int *)malloc(n*sizeof(int));

double j_ceff $=0$;

int right_elt,down_elt,curr_elt;

for $(\mathrm{i}=0 ; \mathrm{i}<\mathrm{n} ; \mathrm{i}++)$

bin_num_cp $[\mathrm{i}]=($ bin_num $[\mathrm{i}]==0) ?-1: 1$;

for $(\mathrm{i}=0 ; \mathrm{i}<\mathrm{m} ; \mathrm{i}++)\{$

for $(j=0 ; j<m ; j++)\{$

right_elt $=i^{*} m+(j+1) \% m$;

down_elt $=((\mathrm{i}+1) \% \mathrm{~m}) * \mathrm{~m}+\mathrm{j}$;

curr_elt $=\mathrm{i} * \mathrm{~m}+\mathrm{j}$;

j_ceff+bin_num_cp[curr_elt]*

(bin_num_cp[right_elt]+bin_num_cp[down_elt]); \}\}

free(bin_num_cp);

return j_ceff; $\}$

static inline void inc_bin_num(int* bin_num, int $\mathrm{n})\{$

int $\mathrm{i}=-1$;

for $(\mathrm{i}=0 ; \mathrm{i}<\mathrm{n} \& \&$ bin_num $[\mathrm{i}] !=0 ; \mathrm{i}++)$ bin_num $[\mathrm{i}]=0$;

if $(\mathrm{i}<\mathrm{n})$

bin_num $[i]=1 ;\}$

static inline int calc_sum(int* bin_num, int $\mathrm{n})\{$

int $\operatorname{sum}=0, i$;

for $(\mathrm{i}=0 ; \mathrm{i}<\mathrm{n} ; \mathrm{i}++)$

sum $+=($ bin_num $[\mathrm{i}]==0 ?-1: 1 ;)$

return sum; $\}$

void dump_results(char $*$ fname, double $* * \mathrm{~h}$, int $\mathrm{n}$, int nceffs $\{$ \{

FILE $* \mathrm{fp}=$ fopen(fname, "w");

int $\mathrm{i}, \mathrm{j}$;

for $(\mathrm{i}=0 ; \mathrm{i}<=2 * \mathrm{n} ; \mathrm{i}+=2)\{$

$$
\text { for }(\mathrm{j}=\text { nceffs }-1 ; \mathrm{j}>0 ; \mathrm{j}--)
$$

fprintf(fp,"A(\%d,\%d) $\% .0 f \backslash t \backslash t \quad x^{\wedge} \% d^{*} y^{\wedge} \% d \backslash n "$, $\mathrm{i} / 2,(2 * \mathrm{n}-(\mathrm{j} * 4-2 * \mathrm{n})) / 2, \mathrm{~h}[\mathrm{i}][\mathrm{j}], 2 * \mathrm{n}-2 *(2 * \mathrm{n}-(\mathrm{j} * 4-$

$2 * n)) / 2, n-i)$;

fprintf(fp,"\n");

fclose(fp); $\}$ 


\section{Appendix B}

In this Appendix, the $\mathrm{C}++$ code for the show-bit algorthim for a square lattice of 16 sites is provided.

\#include $<$ stdio.h $>$

\#include $<$ math.h $>$

\#include $<$ stdlib.h $>$

\# define N 16

\# define $\mathrm{J} 1$

\# define SIZE pow $(2, \mathrm{~N})$

void showbits (int $\mathrm{n}$, int a[]);

int main ()\{

int a[16];

int i,n;

$\mathrm{n}=$ (int)SIZE;

double ht,q,H,lnq,beta, $\mathrm{T}$;

for $(\mathrm{H}=0.0 ; \mathrm{H}<=2.0 ; \mathrm{H}=\mathrm{H}+0.1)\{$

for $(\mathrm{T}=1 ; \mathrm{T}<=5 ; \mathrm{T}=\mathrm{T}+0.1)\{$

beta $=1 / \mathrm{T}$;

$\mathrm{q}=0.0$;

for $(\mathrm{i}=0 ; \mathrm{i}<\mathrm{n} ; \mathrm{i}++)\{$

showbits (i,a);

$\mathrm{ht}=-\mathrm{J} *(\mathrm{a}[0] *(\mathrm{a}[1]+\mathrm{a}[4])+\mathrm{a}[1] *(\mathrm{a}[2]+\mathrm{a}[5])+\mathrm{a}[2] *$

$(\mathrm{a}[3]+\mathrm{a}[6])+\mathrm{a}[3] *(\mathrm{a}[0]+\mathrm{a}[7])+\mathrm{a}[4] *(\mathrm{a}[5]+\mathrm{a}[8])+$

$\mathrm{a}[5] *(\mathrm{a}[6]+\mathrm{a}[9])+\mathrm{a}[6] *(\mathrm{a}[7]+\mathrm{a}[10])+\mathrm{a}[7]^{*}$

$(\mathrm{a}[4]+\mathrm{a}[11])+\mathrm{a}[8] *(\mathrm{a}[9]+\mathrm{a}[12])+\mathrm{a}[9] *(\mathrm{a}[10]+\mathrm{a}[13])+$ $\mathrm{a}[10]^{*}(\mathrm{a}[11]+\mathrm{a}[14])+\mathrm{a}[11]^{*}(\mathrm{a}[8]+\mathrm{a}[15])+\mathrm{a}[12]^{*}$

$(\mathrm{a}[13]+\mathrm{a}[0])+\mathrm{a}[13] *(\mathrm{a}[14]+\mathrm{a}[1])+\mathrm{a}[14]^{*}$

$\left.(\mathrm{a}[15]+\mathrm{a}[2])+\mathrm{a}[15]^{*}(\mathrm{a}[12]+\mathrm{a}[3])\right)$

$-\mathrm{h} *(\mathrm{a}[0]+\mathrm{a}[1]+\mathrm{a}[2]+\mathrm{a}[3]+\mathrm{a}[4]+\mathrm{a}[5]+\mathrm{a}[6]+\mathrm{a}[7]+$

$\mathrm{a}[8]+\mathrm{a}[9]+\mathrm{a}[10]+\mathrm{a}[11]+\mathrm{a}[12]+\mathrm{a}[13]+\mathrm{a}[14]+\mathrm{a}[15])$;

$\mathrm{q}=\mathrm{q}+\exp (-\mathrm{ht} *$ beta $) ;\}$

$\ln q=\log (q)$;

printf("\%f \n",lnq);\}

printf("******************* $\backslash \mathrm{n} ") ;\}\}$

void showbits (int $n$,int a[]) \{

int $\mathrm{i}, \mathrm{k}, \mathrm{p}=0$, andmask;

for $(\mathrm{i}=15 ; \mathrm{i}>=0 ; \mathrm{i}--)\{$

andmask $=1<<\mathrm{i}$;

$\mathrm{k}=\mathrm{n} \&$ andmask;

$\mathrm{k}==0 ? \mathrm{a}[\mathrm{p}]=-1: \mathrm{a}[\mathrm{p}]=1$;

$\mathrm{p}++;\}\}$

\section{References}

1. Chamberlin R V 2000 Nature $\mathbf{4 0 8} 337$

2. Peryard M 2006 Nature Phys. 213
3. Onsager L 1944 Phys. Rev. 65117

4. Lee T D and Yang C N 1952 Phys. Rev. 87410

5. Bragg W L and Williams E J 1934 Proc. R. Soc. A 145 699

6. Bethe H A 1935 Proc. R. Soc. A 150552

7. Domb C and Green M S 1972 Phase transitions and critical phenomena (London: Academic Press)

8. Wilson K G 1971 Phys. Rev. B 43174

9. Kadanoff L P, Goetze W, Hamblen D, Hecht R, Lewis E A S, Palciauskas V V, Rayl M, Swift J, Aspnes D and Kane J 1967 Rev. Mod. Phys. 39395

10. Hill T L 1956 Statistical mechanics - Principles and selected applications (New York: McGraw Hill)

11. McCoy B M and Wu T T 1973 Two-dimensional Ising model (Massachusetts: Harvard University Press)

12. Pushpalatha K and Sangaranarayanan M V 1997 J. Electroanal. Chem. 42539

13. Saradha R and Sangaranarayanan M V 1998 J. Phys. Chem. B 1025468

14. Aoki K 2001 J. Electroanal. Chem. 5131

15. Lenz P, Zagrovic B, Shapiro J and Pande V S 2004 J. Chem. Phys. 1206769

16. Kole P R, De Vries R J, Poelsema B and Zandvliet H J W 2005 Solid State Commun. 136356

17. Thompson C J 1986 Ann. Probab. 141129

18. Plischke M and Bergersen B 1989 Equilibrium statistical physics (New Jersey: Prentice-Hall)

19. Chandler D 1987 Introduction to statistical mechanics (New York: Oxford University Press)

20. Newman M E J and Barkema G T 1999 Monte Carlo methods in statistical physics (Oxford University Press)

21. Kasteleyn P W 1967 Graph theory and crystal physics (New York: Academic Press)

22. Chang S-C and Shrock R 2009 J. Stat. Phys. 138 496

23. Finch S R 2003 Mathematical constants (Cambridge: Cambridge University Press)

24. Dokholyan N V, Li L, Ding F and Shakhnovich E I 2002 Proc. Natl. Acad. Sci. USA 998637

25. Bodroza-Pantic O 2004 J. Math. Chem. 35139

26. Maayan A 2009 J. Biol. Chem. 2845451

27. Temperley H N V and Lieb E M 1971 Proc. R. Soc. Lond. A 322251

28. Huang K 1963 Statistical mechanics (New York: John Wiley \& Sons)

29. Kramers H A and Wannier G H 1941 Phys. Rev. 60 252

30. Nandhini G and Sangaranarayanan M V 2009 J. Chem. Sci. 121595

31. Noor F and Morgera S D 1992 IEEE Trans. Signal Process. 402093

32. Bellman R 1997 Introduction to matrix analysis (USA: Society for Industrial and Applied Mathematics)

33. Landau D P 1976 Phys. Rev. B 132297 